\title{
Secondary vestibulocerebellar projections to the flocculus and uvulo-nodular lobule of the rabbit: a study using HRP and double fluorescent tracer techniques
}

\author{
A.H. Epema, N.M. Gerrits, and J. Voogd \\ Department of Anatomy, HB 13, Erasmus University Rotterdam, P.O. Box 1738, 3000 DR Rotterdam, The Netherlands
}

\begin{abstract}
Summary. The distribution of vestibular neurons projecting to the flocculus and the nodulus and uvula of the caudal vermis (Larsell's lobules X and IX) was investigated with retrograde axonal transport of horseradish peroxidase and the fluorescent tracers Fast Blue, Nuclear Yellow and Diamidino Yellow. The presence of collateral axons innervating the flocculus on one hand and the nodulus and uvula on the other was studied with simultaneous injection of the different fluorescent tracers. The distribution of vestibular neurons projecting to either flocculus or caudal vermis is rather similar and has a bilateral symmetry. The projection from the magnocellular medial vestibular nucleus is very sparse, while that from the lateral vestibular nucleus is absent. The majority of labeled neurons was found in the medial, superior, and descending vestibular nuclei, in that order. Double labeled neurons were distributed in a similar way as the single labeled ones. Labeled neurons project to the nodulus and uvula, the flocculus, and to both parts of the cerebellum simultaneously in a ratio of $12: 4: 1$. Five different populations of vestibulocerebellar neurons can be distinguished on the basis of their projection to the: (1) ipsilateral flocculus, (2) contralateral flocculus, (3) ipsilateral flocculus and nodulus/uvula, (4) contralateral flocculus and nodulus/uvula, and (5) nodulus/uvula.
\end{abstract}

* Present address: Dept. of Anaesthesiology, University Hospital Leiden, Rÿnsburgerweg 10, 2333 AL Leiden, The Netherlands

Abbreviations. bc brachium conjunctivum; CE external cuneate nucleus; cr restiform body; CO cochlear nuclei; DV descending vestibular nucleus; $F$ fastigial nucleus; FL flocculus; flm medial longitudinal fascicle; gV vestibular ganglion; gVII facial genu; IN interstitial nucleus of the eight nerve; LV lateral vestibular nucleus; MVc caudal medial vestibular nucleus; MVmc magnocellular medial vestibular nucleus; $\mathrm{MVpc}$ parvocellular medial vestibular nucleus; NVpar parabrachial vestibular nucleus; nVII facial nerve; $\mathrm{PH}$ prepositus hypoglossal nucleus; $\mathrm{rV}$ descending root of the trigeminal nerve; $\mathrm{S}$ solitary tract and nucleus; sad dorsal acustic striae; SV superior vestibular nucleus; $X$ group $X$; $Y$ group $Y$; VI abducens nucleus.

Offprint requests to: N.M. Gerrits (address see above)
Key words: Vestibular nuclei - Mossy fiber - Cerebellum - Flocculus - Uvula - Nodulus - Fluorescent tracer Rabbit

\section{Introduction}

The vestibular nuclei are among the many sources of cerebellar mossy fibers (Brodal 1974). This "secondary vestibulocerebellar" connection (Dow 1936), as opposed to the primary vestibulocerebellar afferents from Scarpa's ganglion, has been subjected to a number of investigations employing the method of retrograde transport of horseradish peroxidase (HRP). These studies included a variety of species such as the rat (Blanks et al. 1983), the rabbit (Alley et al. 1975; Yamamoto 1979), the cat (Gould 1980; Kotchabhakdi and Walberg 1978; Sato et al. 1983), and the macaque monkey (Brodal and Brodal 1985; Langer et al. 1985). Together with studies using anterograde axonal tracing (Magras and Voogd 1985; Thunnissen et al. 1989), they have demonstrated that the flocculus and the uvulo-nodular lobule are the main recipients of secondary vestibulocerebellar mossy fibers. The data obtained with the anterograde and retrograde techniques show that the neurons projecting to these lobules are not arranged in topographically separated populations within the vestibular complex.

Flocculus, uvula and nodulus, the constituents of the "classical" vestibulocerebellum, have been implicated in different functions. According to Ito (1984) the flocculus has a modulatory effect on the vestibulo-ocular reflex and its adaptive properties. The nodulus and the uvula of the posterior vermis influence the vestibulo-collic and postural reflexes (Precht 1979).

In view of this functional specialization, the question arises whether vestibular mossy fibers to the flocculus and the uvulo-nodular lobule are collaterals from single axons or originate from separate neurons. In the present study this problem was investigated using simultaneous 
injection of different retrogradely transported fluorescent tracers: Fast Blue and Nuclear Yellow or Diamidino Yellow (Kuypers et al. 1980). Since knowledge of the secondary vestibulocerebellar projection in the rabbit is limited, and restricted to the connection with the flocculus (Alley et al. 1975; Yamamoto 1979), the distribution of vestibulocerebellar neurons was also determined in separate experiments with injections of HRP in the flocculus and uvulo-nodular lobule.

\section{Experimental procedures}

The experiments were carried out on 11 pigmented Dutch belted rabbits (body weight $2-2.5 \mathrm{~kg}$ ). They received a premedication with Hypnorm $(0.3 \mathrm{ml} / \mathrm{kg})$ and atropine $(0.05 \mathrm{mg} / \mathrm{kg})$, and were placed in a stereotaxic frame with a rabbit head holder. General anaesthesia was maintained with a halothane-oxygen mixture administered through an endotracheal tube. The cerebellar caudal vermis was reached by a dorsal approach via an enlargement of the occipital foramen. Injections into the right flocculus were made following its exposure from the middle cranial fossa by removing the occipital lobe and incision of the tentorium cerebelli.

\section{HRP procedure}

Pressure injections of HRP $(0.5-0.8 \mu \mathrm{l}$; Sigma type VI or Boeringer grade II $33 \%$ in phosphate buffered saline) were made using a $1 \mu 1$ Hamilton syringe with a 25 gauge needle or with a micropipette (tip diameter $25-30 \mu \mathrm{m}$ ) connected to a hydraulic system, at the rate of $0.1 \mu \mathrm{l}$ per $10 \mathrm{~min}$. To reduce unwanted spread of HRP, delivery started 5 min after placing the needle or pipette in position; they were removed 10 to $15 \mathrm{~min}$ after the injection. After survival times of 1-2 days (Table 1) the animals were killed with an overdose of Nembutal, heparinized and transcardially perfused with 0.51 saline, 1.51 citrate buffer $(0.1 \mathrm{M}, \mathrm{pH} 7.2)$ containing $1 \%$ formaldehyde and $1.25 \%$ glutaraldehyde and washed with 0.51 citrate buffer $(0.1 \mathrm{M}$, pH 7.2) containing 8\% sucrose (procedure II of Rosene and Mesulam 1978). The brains were embedded in $10 \%$ gelatin (Voogd and Feirabend 1981) and transversely sectioned at $40 \mu \mathrm{m}$ on a freezing microtome. Alternate sections were incubated with diaminobenzidine (DAB) and tetramethylbenzidine (TMB) (Mesulam 1982) and counterstained with Cresyl Violet or Neutral Red. Sections were coverslipped with Permount. Cells were considered as

Table 1. Listing of experiments with injections in the flocculus (FL) and uvulo-nodular lobule (IX +X). HRP: $33 \%$ horseradish peroxidase; DY: $2 \%$ Diamidino Yellow; FB: 3\% Fast blue; NY: $1 \%$ Nuclear Yellow. Injected volumes in $\mu 1$, survival time in days

\begin{tabular}{|c|c|c|c|c|}
\hline \multirow[t]{2}{*}{$\operatorname{Exp}$} & \multicolumn{2}{|l|}{ FL } & \multicolumn{2}{|l|}{$\mathrm{IX}+\mathrm{X}$} \\
\hline & vol & surv & vol & surv \\
\hline K26 & $0.5 \mathrm{HRP}$ & 2.0 & & \\
\hline C562 & 0.8 HRP & 1.1 & & \\
\hline C588 & & & $0.8 \mathrm{HRP}$ & 2.0 \\
\hline C1031 & & & $0.5 \mathrm{HRP}$ & 2.0 \\
\hline K 74 & 0.7 FB & 8 & $0.7 \mathrm{DY}$ & 6 \\
\hline K 75 & 0.9 FB & 7 & $0.9 \mathrm{DY}$ & 4 \\
\hline $\mathrm{K} 78$ & $0.9 \mathrm{FB}$ & 10 & $0.9 \mathrm{DY}$ & 6 \\
\hline K 275 & $0.8 \mathrm{FB}$ & 19 & $0.8 \mathrm{DY}$ & 11 \\
\hline $\mathrm{C} 825$ & $0.5 \mathrm{NY}$ & 0.8 & $0.9 \mathrm{FB}$ & 12 \\
\hline $\mathrm{C} 858$ & $0.5 \mathrm{NY}$ & 0.9 & $0.7 \mathrm{FB}$ & 12 \\
\hline C1275 & $0.2 \mathrm{DY}$ & 6 & $0.4 \mathrm{FB}$ & 14 \\
\hline
\end{tabular}

HRP-labeled (Fig. 1D-F) when they met the criteria described by Nauta et al. (1974).

\section{Fluorescent tracer procedure}

Pressure injections of Fast Blue (FB) (Dr. Illing, KG; $3 \%$ in distilled water; Bentivoglio et al. 1980b), Nuclear Yellow (NY) (Hoechst; $1 \%$ in distilled water containing $2 \%$ dimethylsulfoxide; Huisman et al. 1982) and Diamino Yellow (DY) (Dr. Illing, $\mathrm{KG} ; 2 \%$ in distilled water; Keizer et al. 1983) were made with a micropipette (tipdiameter 25-50 $\mu \mathrm{m}$ ) attached to a hydraulic system, and in volumes from 0.2-0.9 $\mu$ l (Table 1).

Migration of NY out of the retrogradely labeled neurons into glial cells (Bentivoglio et al. 1980a) could be avoided by using survival times of less than one day, as established in preliminary experiments. Animals receiving fluorescent tracer injections were operated twice because the optimal survival time for the two tracers differed (Table 1).

The animals were killed with an overdose of Nembutal, heparinized and transcardially perfused with 1.51 saline $(2.7 \% \mathrm{NaCl})$ followed by 21 citrate buffer $(0.1 \mathrm{M}, \mathrm{pH} 7.2)$, containing $30 \%$ formaldehyde and washed with 11 citrate buffer $(0.1 \mathrm{M}, \mathrm{pH} 7.2$ ) containing $8 \%$ sucrose. Except for the experiments in which NY was injected, the brains were embedded in $10 \%$ gelatin (Voogd and Feirabend 1981), and transversely sectioned at $30 \mu \mathrm{m}$ on a freezing microtome. One out of four sections was immediately mounted from distilled water and air-dried at room temperature in the dark. Sections were stored in light tight boxes at $4^{\circ} \mathrm{C}$. The presence of fluorescent cells was studied with a Zeiss Ploemopack fluorescence microscope equipped with a filter-mirror system providing an excitation wave length of $360 \mathrm{~nm}$. Single and double labeled neurons (Fig. 1A-C) showed characteristics matching previously published data (Bentivoglio et al. 1980a; Keizer et al. 1983; Kuypers et al. 1980). The differentiation of the injection sites into zones I-III was according to the same authors. Overlap of the zones III of injections in the flocculus and caudal vermis did not occur in the central white matter of the cerebellum. Experiments with leakage of tracer into the ventricular system were excluded.

The distribution of labeled neurons was plotted on an $\mathrm{X}-\mathrm{Y}$ recorder (Hewlett Packard) connected to displacement transducers attached to the stage of the Zeiss fluorescence microscope. Fluorescent tracer sections were counterstained with Cresyl Violet subsequent to the mapping to study the localization of labeled neurons and the architecture of the vestibular nuclei and the cerebellum.

The nomenclature of the cerebellum follows the description of Van Rossum (1969), Larsell (1970) and Yamamoto (1979). The vestibular nuclei were subdivided according to Epema et al. (1988). To facilitate comparison of the distribution of labeled neurons in the different experiments they were plotted in horizontal diagrams of the vestibular nuclei, prepared from the charted sections by orthogonal projection of the boundaries of the nuclei (Epema et al. 1988).

\section{Results}

By creating two groups of experiments in which the specific fluorescent tracers were exchanged with respect to the injection site, an attempt was made to counteract possible effects of differences in transport capacity of the dyes. From either group one experiment was choosen for illustration. To obtain maximum uptake of the fluorescent tracers, the injections were intentionally large and in case of the uvulo-nodular lobule mostly bilateral. The distribution of vestibulocerebellar neurons labeled with fluorescent tracers was also compared with a number of 

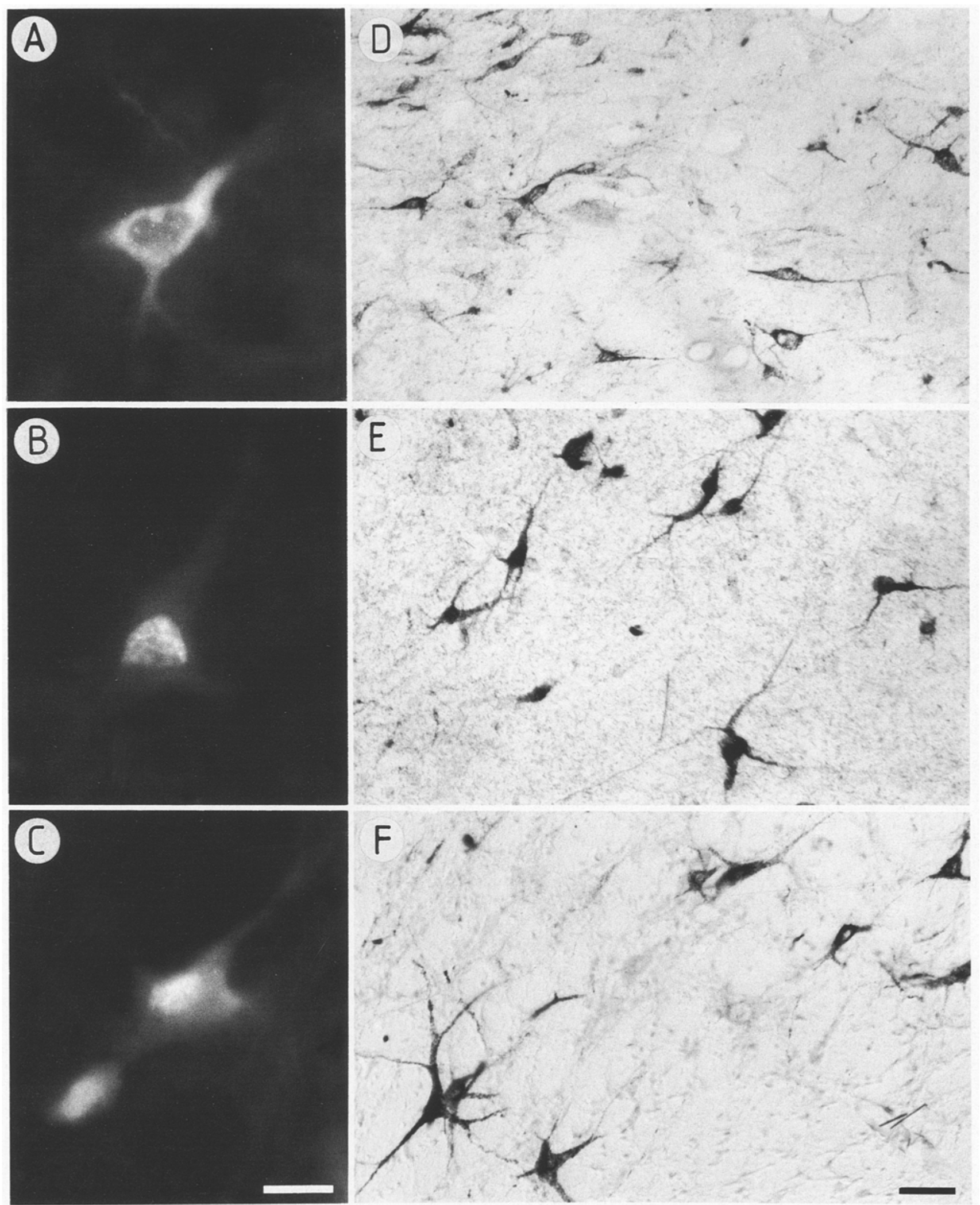

Fig. 1A-F. Neurons retrogradely labeled with fluorescent tracer. A Fast Blue (FB); B diamidino Yellow (DY), double labeled with FB + DY. Scale bar for $\mathbf{A}-\mathbf{C}$ represents $20 \mu$. Vestibulocerebellar neurons labeled retrogradely with HRP; $\mathbf{D}$ superior vestibular nucleus; $\mathbf{E}$ caudal medial vestibular nucleus; $\mathbf{F}$ descending vestibular nucleus. Scale bar for $\mathbf{D}-\mathbf{F}$ represents $50 \mu$

Fig. 2. Injections in the right flocculus. Extent of injection sites C562 (HRP), K26 (HRP) (DAB reaction), C825 (NY), K78 (FB) (zone I: black, zone II: dark hatching; zone III: light hatching) in transverse sections through their largest extent and projected onto the floccular surface (according to Yamamoto 1979) (top right). Distribution of labeled neurons in C562 in transverse sections (left). Diagram of the vestibular nuclei showing the overall distribution of labeled neurons in C562 and K26 (bottom right). Left and right side of the brain as illustrated 

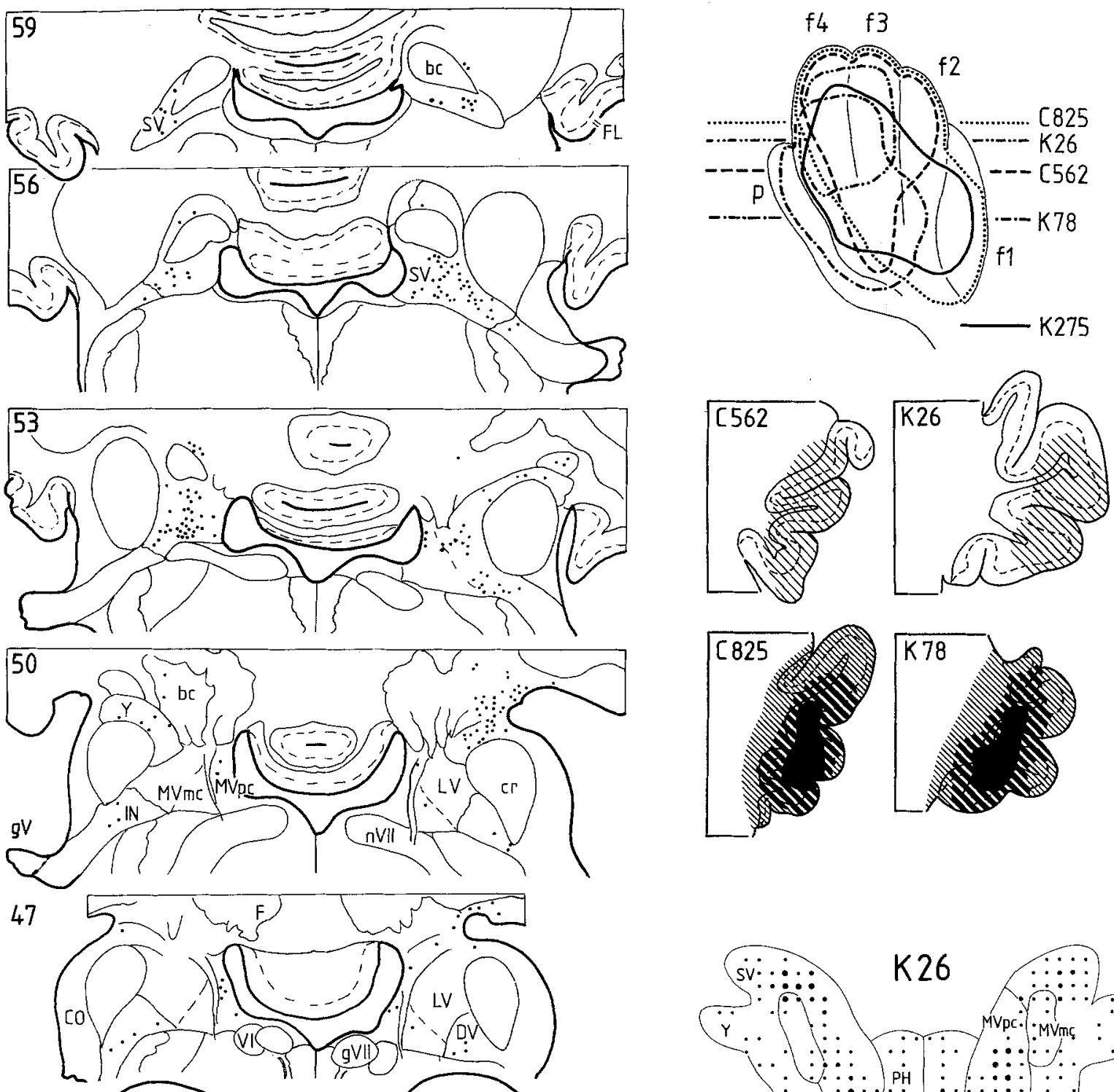

41

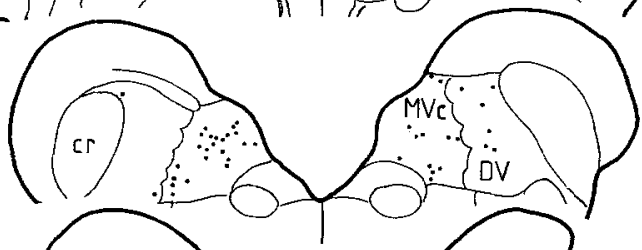

38

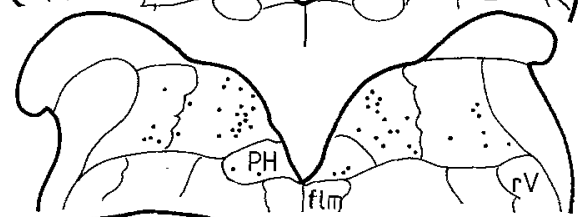

35

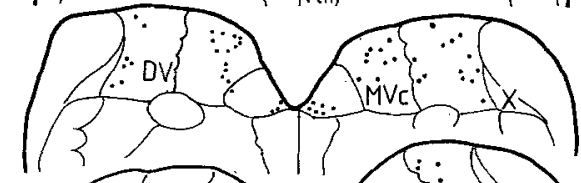

33
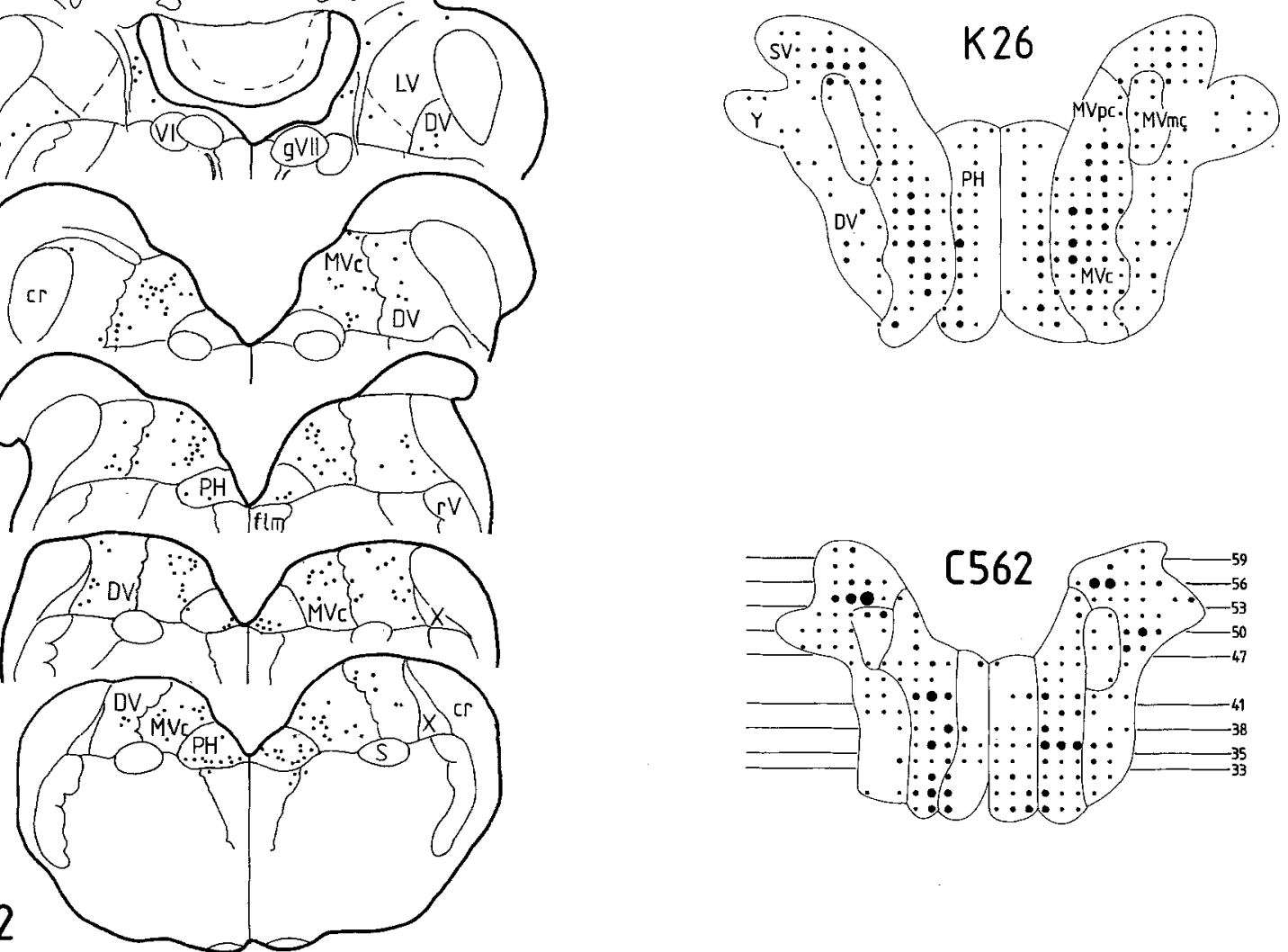

Fig. 2. 
cases with injection of HRP into the flocculus and uvulonodular lobule to detect tracer-specific differences in uptake or transport.

\section{Injections in the flocculus}

The injection sites of the HRP experiments K26 and C562 involved the rostral flocculus and included to differing degrees Yamamoto's (1979) folia f2, f3 and f4 (Fig. 2). The injections did not involve the brachium pontis or the central cerebellar white matter. In experiment C825 the zones I and II of the NY injection site in the flocculus covered part of folia $\mathrm{f} 1$ and $\mathrm{f} 4$ and most of folia $\mathrm{f} 2$ and $\mathrm{f} 3$. In experiment $\mathrm{K} 78$ the FB injection site was smaller and the zones I and II included folia f3, f4 and $p$, leaving the rostral part of the folia free of tracer. The FB injection site of experiment K275 had a similar rostrocaudal position as $\mathrm{K} 78$, but included folia $\mathrm{fl}-\mathrm{f} 4$. Zones I and II of the DY injection site of experiment C1275 (not illustrated) included the rostral three-fourth of folium f4. In all cases zone III extended up to the pial surface and included the medially located floccular white matter (Fig. 2). Further medialward diffusion of tracer seemed effectively blocked by the brachium pontis. Thus, the central cerebellar nuclei and group y were well outside the labeled area.

In all cases labeled neurons were present bilaterally, throughout the vestibular subnuclei, but absent from the lateral vestibular nucleus (LV), group $x$ and the vestibular ganglion (Figs. 2, 4, 5). In the HRP experiments differences in laterality were small; not more than a few percent. In the fluorescent tracer experiments the number of labeled cells at the ipsilateral side was higher in two cases $(\mathrm{C} 825, \mathrm{~K} 78,25 \%)$ and lower in the other two cases (C1275, 8\%; K275, 25\%) (Table 2).

In the superior vestibular nucleus (SV), labeled cells are most abundant in the centre and scarce in the periphery of the nucleus. There is no clear interruption between the areas with labeled neurons in the SV and the labeling in surrounding subnuclei such as the parabrachial vestibular nucleus (NVpar), the interstitial nucleus of the eighth nerve (IN) and group y. A small amount of labeled cells was always found in the centre and dorsal parts of the parvocellular medial vestibular nucleus (MVpc). Very few cells were observed in the magnocellular medial vestibular nucleus $(\mathrm{MVmc})$.

In the caudal aspect of the vestibular complex, labeled neurons are distributed throughout the rostrocaudal extent of the caudal medial vestibular $(\mathrm{MVc})$ and the descending vestibular (DV) nuclei. Rostrally, the area with labeling seems continuous with that in the MVpc. In the MVc most labeled cells are present in the central part of the nucleus. Most labeled neurons in the DV were located laterally in the nucleus, and clearly separated from the labeling in the MVc. The majority of labeled neurons in the prepositus hypoglossal nucleus (PH) is present ventrally in its caudal half. A substantial number of labeled cells is present in the adjacent dorsal aspect of the medial longitudinal fascicle (flm) (Fig. 2).

\section{Injections in the uvulo-nodular lobule}

In experiment C588, the HRP injection site covers most of the sublobules IXc-d on the right side as well as the medial one-third of these lobules on the left side (Fig. 3). The injection in case C1031 is bilateral and includes most of the white matter at the base of lobules IX and X (Fig. 3 ), thereby interrupting the connections of the central two-third of their expanse of cortex. In experiment C825 the FB fluorescent zones I-III covered bilaterally the medial part of the lobules X and IXd and the ventral aspect of lobule IXc. The injection site in the FB experiment $\mathrm{C} 1275$ (not illustrated) matched the extent of case C825 in the right hemivermis, but on the left side included only the medial one-third of folium IXd. The zones I to III in the DY injection site of experiment K78 mainly included the medial part of the folia IXc-d and the ventral part of IXb, but zone II penetrated more deeply into the white matter at the base of the lobules, including the basis of lobule X. The DY injection site of experiment K275 is unilateral (Fig. 3). It only involves the centre of lobule IXd and the apical half of lobule X (Fig. 3). The injection sites all include a substantial proportion of the lobules IXd and $X$, which were shown by Thunnissen et al. (1989) to contain the majority of vestibulocerebellar mossy fiber terminals.

In all experiments, labeled neurons were found bilaterally throughout the vestibular complex, but were not observed in the LV or the infracerebellar nucleus (Figs. $3-5$ ). A large number of labeled neurons was always present in the vestibular ganglion and in group $\mathrm{X}$, lateral to the caudal part of the DV. Differences in laterality were smaller than in the cases with injections in the flocculus. The number of labeled cells was higher in the ipsilateral ( $=$ side of the flocculus injection) vestibular nuclei in two cases $(\mathrm{K} 78,4 \% ; \mathrm{C} 1275,17 \%)$ and lower in two cases (C825, 3\%; K275, 19\%) (Table 2). The number of uvulo-nodular projection cells in C825 and K78 (Table 2) differs by a factor 2 and represent the extremes of the series of experiments. However, they are in the same range as the data obtained with HRP. From the present material and also from fluorescent tracer injections elswhere in the cerebellum we have the impression that the number of labeled precerebellar neurons strongly depends on the extent of damage to the white matter inflicted in zone I.

In the SV, labeled cells showed a tendency to be located in the central and medial parts of the nucleus. This area with labeled neurons continues into the NVpar and group y, but their number is considerably smaller there. Labeling in the medial vestibular nucleus is concentrated in the dorsal part of the MVpc and shifts to a more central position in the $\mathrm{MVc}$, forming a continuous area of labeled cells. The number of labeled neurons in the MVmc was small. The rostral part of the DV contained a limited number of labeled cells. At caudal levels of the DV they were more abundant and not clearly separated from the labeling in MVc and group $\mathrm{x}$. The high number of labeled neurons caudally in the DV, the $\mathrm{MVc}$ and group $\mathrm{x}$ in the experiments with fluorescent 

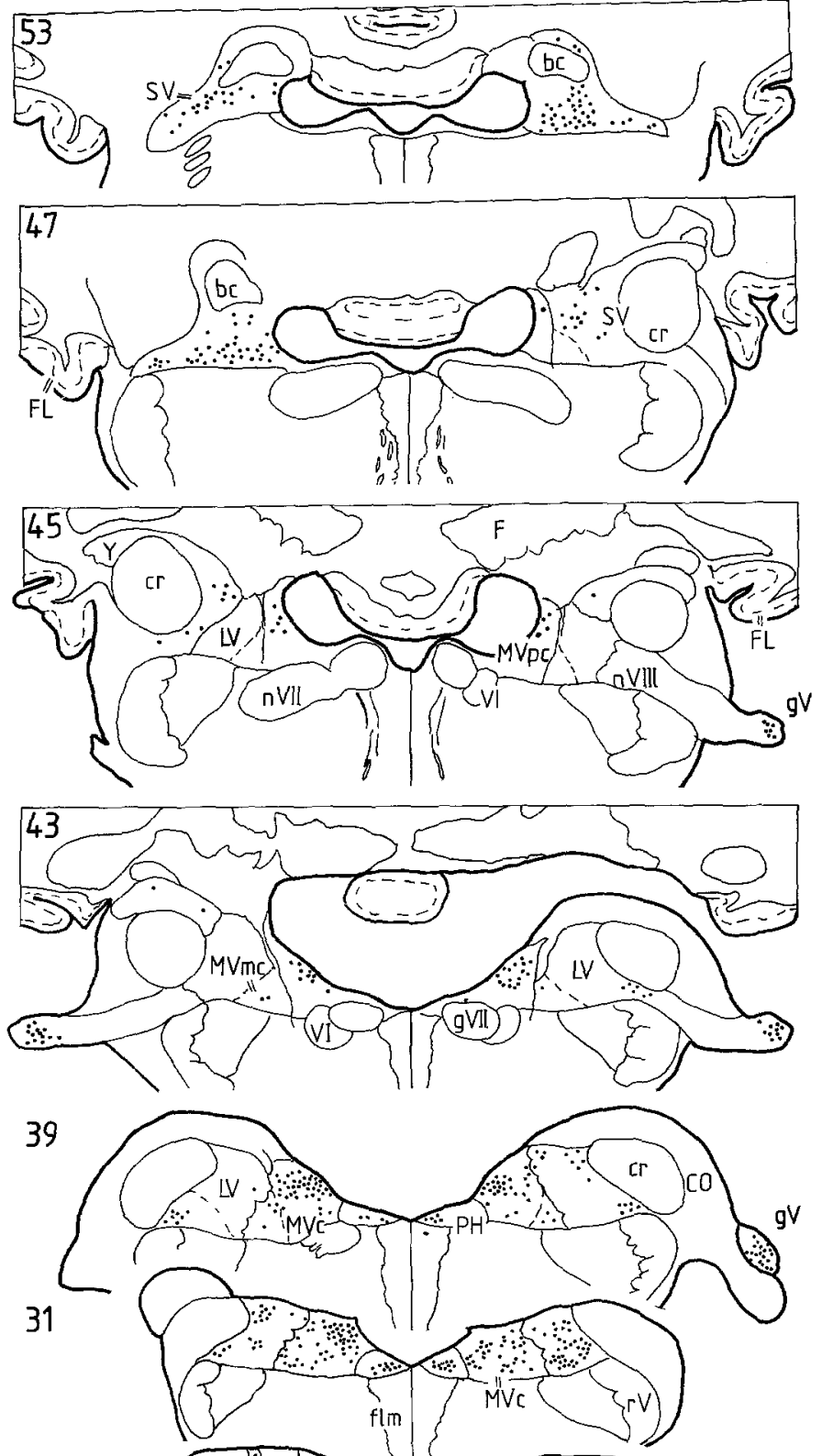

27

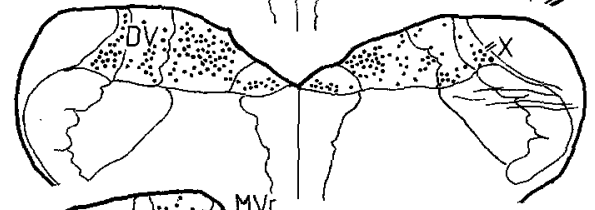

21

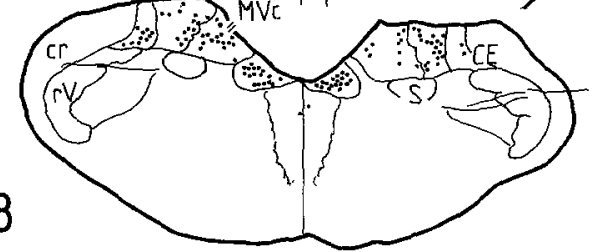

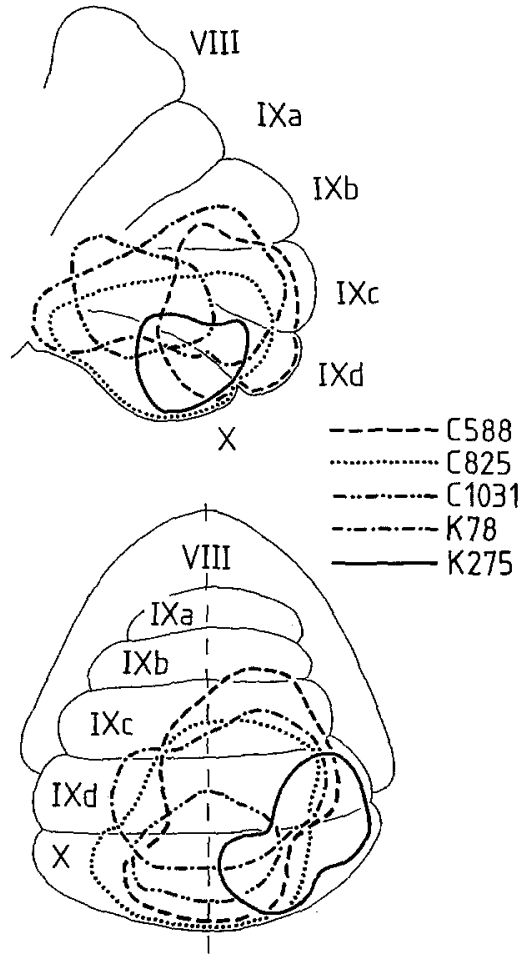
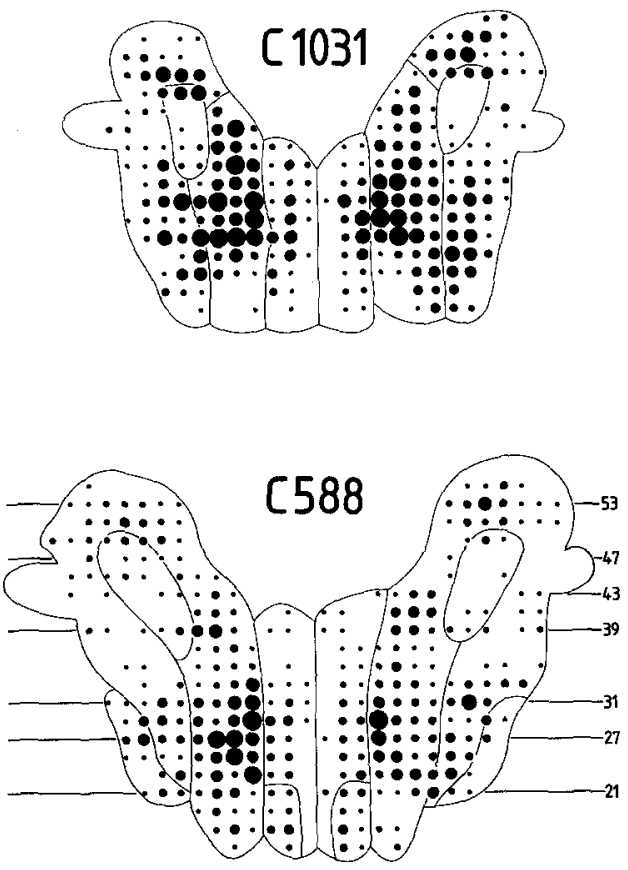

Fig. 3. Injections in the uvulo-nodular lobule. Extent of injection sites C588 (HRP), C1031 (HRP) (DAB reaction), C825 (FB), K78 (DY) (boundary of zone II) projected onto the cerebellar posterior surface and onto the midsagittal plane (top right). Distribution of labeled neurons in C588 in transverse sections (left). Diagrams of the vestibular nuclei showing the overall distribution of labeled neurons in C588 and $\mathrm{C} 1031$ (bottom right). Left and right side of the brain as illustrated 

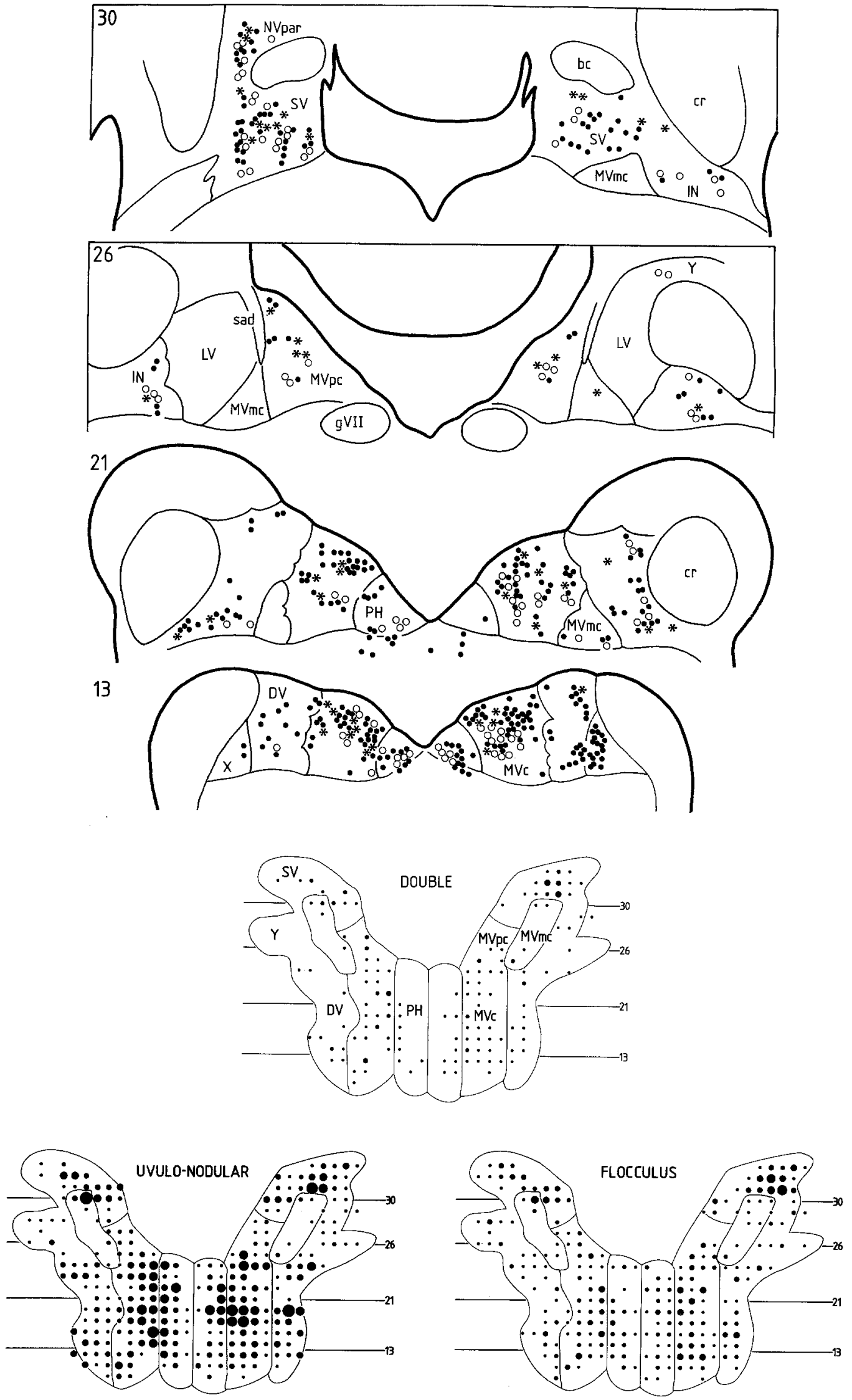

Fig. 4. Experiment C825: injection of $\mathrm{NY}$ into the right flocculus and FB into the uvulo-nodular lobule. Transverse sections (top) through the vestibular complex. Symbols represent labeled neurons; dots: FB, circles: NY, asterisks: double labeled. Diagrams (bottom) illustrate the distribution of single and double labeled neurons projecting to the flocculus, the uvulo-nodular lobule and both areas simultaneously. Left and right side of the brain as illustrated 

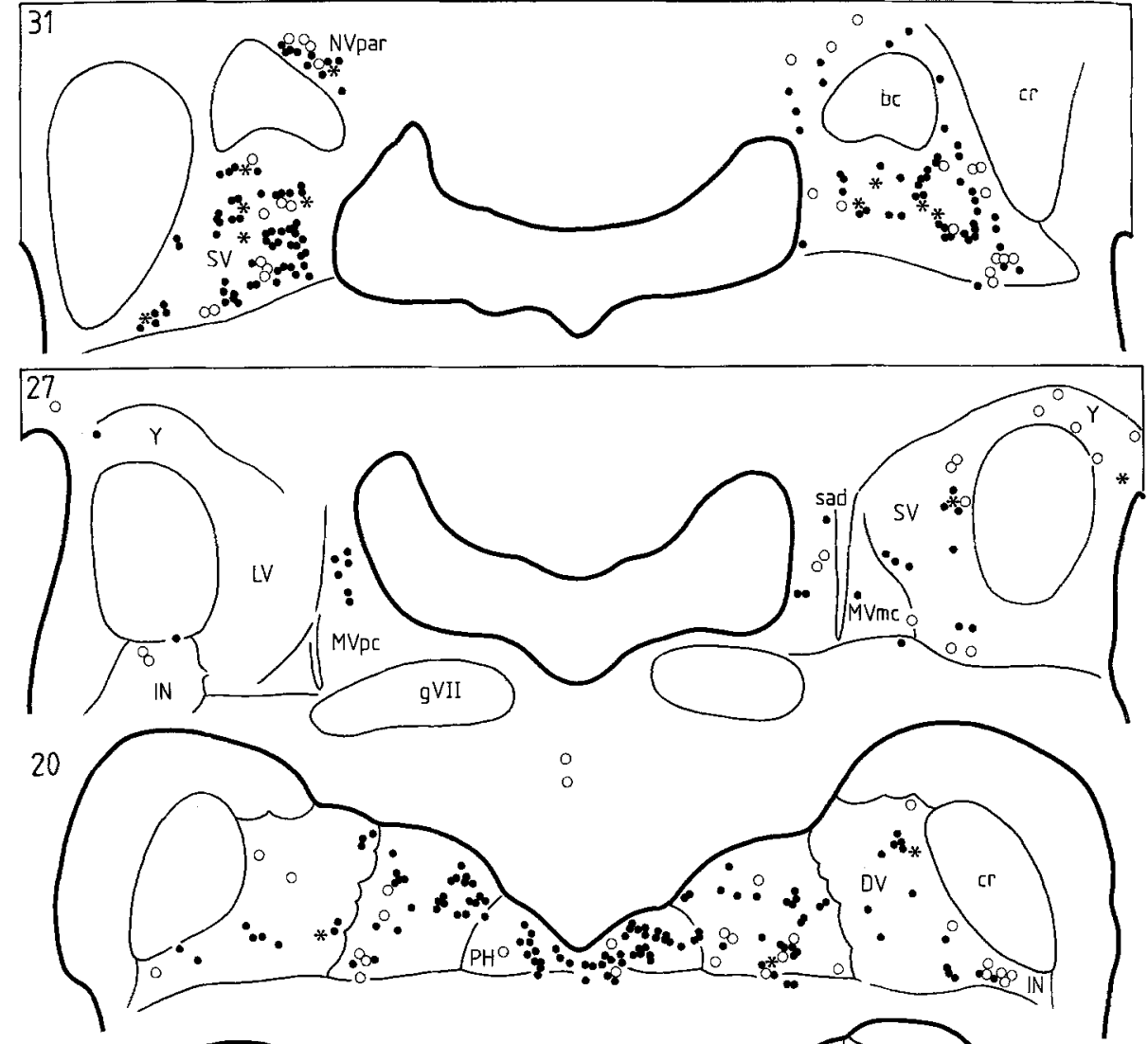

14
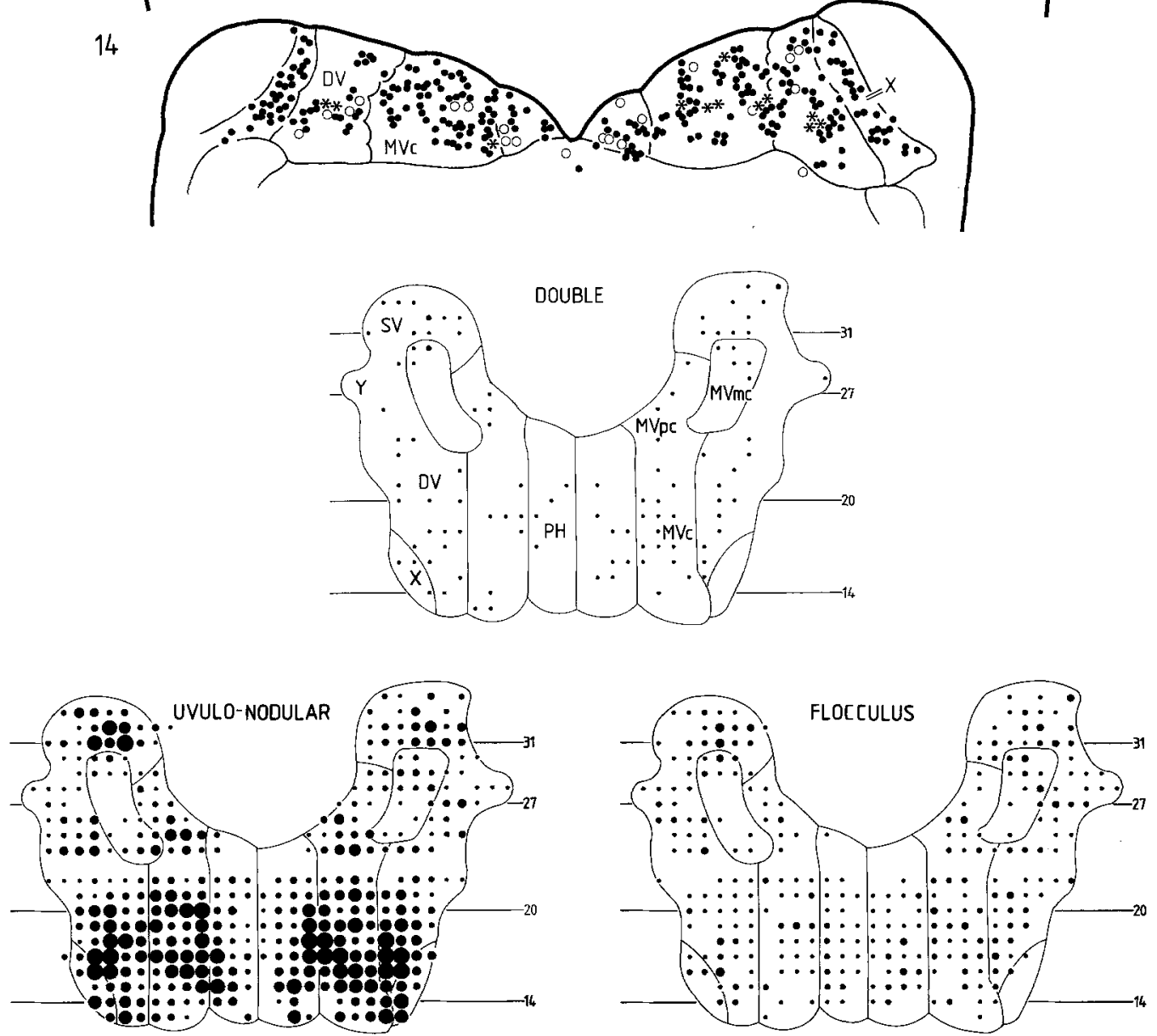

Fig. 5. Experiment K78: injection of FB into the right flocculus and DY into the uvulo-nodular lobule. Transverse sections (top) through the vestibular complex. Symbols represent labeled neurons; dots: DY, circles: FB, asterisks: double labeled. Diagrams (bottom) illustrate the distribution of single and double labeled neurons projecting to the flocculus, the uvulo-nodular lobule and both areas simultaneously. Left and right side of the brain as illustrated 
Table 2. Number of labeled neurons following injection of tracer in uvulo-nodular lobule and/or flocculus, counted in $\mathrm{N}$ equally spaced sections. In experiments C825, C1275, K78 and K275 the section thickness was $30 \mu \mathrm{m}$ and ipsi- and contralateral refers to the right (flocculus injection site) and left sides of the brain respectively. In experiments K26, C562, C588 and C1031 the section thickness was $40 \mu \mathrm{m}$ and ipsi- and contralateral refers to respectively the right (flocculus injection site) and left sides of the brain. The percentages give the sum of ipsi- and contralateral sides as a proportion of the total number of labeled neurons

\begin{tabular}{|c|c|c|c|c|c|c|c|c|c|c|}
\hline \multirow[t]{2}{*}{ Exp } & \multirow[t]{2}{*}{$\mathrm{N}$} & \multicolumn{3}{|c|}{ U-NL single } & \multicolumn{3}{|c|}{ Double } & \multicolumn{3}{|c|}{ FL single } \\
\hline & & contra & ipsi & $\%$ & contra & ipsi & $\%$ & contra & ipsi & $\%$ \\
\hline $\mathrm{K} 78$ & (21) & 1220 & 1258 & 79 & 65 & 64 & 4 & 224 & 288 & 17 \\
\hline K275 & (24) & 632 & 516 & 58 & 26 & 11 & 2 & 460 & 347 & 40 \\
\hline C1275 & (7) & 248 & 296 & 62 & 24 & 20 & 5 & 148 & 136 & 33 \\
\hline C588 & (21) & 897 & 831 & & & & & & & \\
\hline C1031 & (17) & 1020 & 1083 & & & & & & & \\
\hline
\end{tabular}

tracer injections as compared to the HRP experiments may be due to the greater mediolateral extent of the injection sites in these cases. Within the PH, labeled cells occupied a central position throughout its rostrocaudal extent.

\section{Double fluorescent labeled neurons}

Double labeled neurons were found in nearly all subdivisions of the vestibular nuclei as well as in the PH, but were absent from the LV. Their distribution is very similar to that of neurons projecting to the flocculus (Figs. $2,4,5$ ). Consequently they are absent from group $\mathrm{x}$, almost absent from the MVmc and not numerous in the caudal aspect of the vestibular complex where the majority of uvulo-nodular projection neurons are located (Figs. 3-5).

Similar to what was observed with single labeled neurons, the difference in laterality was not pronounced (Table 2). The quantitative differences between C 825 on the one hand and $\mathrm{C} 1275, \mathrm{~K} 78$ and K275 on the other are mainly caused by a different number of labeled neurons in the SV that might be related to the extensive coverage of the rostral folia of the FL with tracer in case C825. The unilateral nature of the caudal vermis injection in K275 may explain the very low number of double labeled neurons in that case.

\section{Discussion}

This study is the first to show the distribution of vestibulocerebellar neurons projecting to the uvula and nodulus of the caudal vermis in the rabbit. It also confirms and extends previous data for vestibular neurons projecting to the flocculus (Alley et al. 1975; Yamamoto 1979). The differences which were observed in the amount of single and double labeling, as well as the distribution of the neurons did not exceed the expectations due to differences in the size and localization of the injection sites. There were no consistent differences between the two groups in which the specific tracers were switched with respect to the injection sites. The different types of neurons were not found in spatially segregated subpopulations, but were intermingled without apparent organization.

Double labeling of neurons can be caused by leakage of tracer out of retrogradely labeled somata followed by successive uptake in glial cells and transfer to other neurons (Bentivoglio et al. 1980a). The probability of leakage of NY in our experiments was reduced by determining optimal survival times in a preliminary series of experiments, which confirmed earlier findings (Bentivoglio et al. 1980a; Kuypers et al. 1980). Following injection with DY no evidence for leakage into glia was found. Since in all experiments single labeled FB and DY or NY neurons were present in (sometimes very) close proximity, leakage is thought to be practically absent.

\section{Neurons projecting to the flocculus}

The origin of the secondary vestibulocerebellar projection to the flocculus has been determined in a number of studies in various mammals using HRP as a retrograde tracer. The two reports on this projection in the rabbit (Alley et al. 1975; Yamamoto 1979) provide little detail about the distribution of labeled neurons. In general, our results are similar to previous data obtained in the rat (Blanks et al. 1983), the cat (Gould 1980; Kotchabhakdi and Walberg 1978; Sato et al. 1983), the bushbaby (Rubertone and Haines 1981) and the macaque (Brodal and Brodal 1985; Langer et al. 1985). Labeled neurons are present bilaterally throughout the $\mathrm{PH}$ and all subdivisions of the vestibular complex, except for the LV. The neurons are most numerous in the MVc, the SV and the $\mathrm{PH}$. According to Gerrits (1985) the SV projects most heavily to the rostral FL. This may explain the high number of retrogradely labeled neurons in this nucleus in case C825. Labeled neurons in the NVpar were not mentioned by previous authors, since this subdivision of the SV was introduced recently (Epema et al. 1988). Labeled vestibulocerebellar neurons showed a tendency to cluster, although not as strongly as illustrated by 
Yamamoto (1979). The difference with reports claiming labeling in the LV can be explained either by the fact that the borders of the LV with the DV and SV are difficult to establish (Kotchabhakdi and Walberg 1978; Langer et al. 1985) or that the MVmc, which only contains small labeled neurons, is sometimes included in the LV (Blanks et al. 1983; Sato et al. 1983). A most striking difference seems to exist between the rat and other animals (rabbit, cat, monkey) with respect to group $x$, which in the rat was found to be densely labeled following injections in the flocculus (Blanks et al. 1983). A species difference cannot be excluded, but another possibility is that the labeled neurons instead of belonging to group $x$ are located in the caudolateral part of the DV, an area which was reported to contain vestibulothalamic projection neurons (Kevetter et al. 1982). A further difference is the exclusively ipsilateral labeling of group $y$ neurons in the rat (Blanks et al. 1983), which were consistently labeled bilaterally in the other species.

The location of labeled neurons in the periphery (mainly ventrally) of the $\mathrm{PH}$ is in agreement with previous reports (Kotchabhakdi et al. 1978; Sato et al. 1983; Yamamoto 1979). An explanation for the markedly lower amount of labeled neurons in the $\mathrm{PH}$ in our study as compared to other studies may be due to the fact that in these cases the injection sites included parts of the ventral paraflocculus (Blanks et al. 1983; Sato et al. 1983).

\section{Neurons projecting to the uvulo-nodular lobule}

The secondary vestibular projection to the lobules IX and $X$ of the posterior vermis has its origin in all subdivisions of the vestibular nuclei except for the LV, and in addition in the $\mathrm{PH}$ and group $\mathrm{x}$. Labeled neurons were present bilaterally in equal numbers, which was also observed following unilateral HRP injections in lobules IX and X (Thunnissen et al. 1989). The greatest density of neurons was observed in the caudal half of the vestibular complex in the MVc, the neighbouring DV and in group $x$, and rostrally in the SV. Our data are in agreement with the results of previous studies in the cat (Gould 1980; Kotchabhakdi and Walberg 1978; Walberg and Dietrichs 1988) and the macaque (Brodal and Brodal 1985). Apart from the difference with respect to group $x$, the neurons which project to either the flocculus or the uvulo-nodular lobule do not occupy separate territories in the vestibular complex.

The projection of the PH to the uvulo-nodular lobule originates from neurons clustered in the centre of this nucleus. This is in agreement with previous data in cat and monkey (Brodal and Brodal 1985; Kotchabhakdi et al. 1978).

\section{Double fluorescent labeled neurons}

Until now, collateral branches heading in the direction of the flocculus and uvulo-nodular lobule have only been reported for some vestibulocerebellar axons (Highstein et al. 1987; Mitsacos et al. 1983). However, these authors were not able to trace the axons to their final destination, due to the limitations of the intra-axonal HRP staining technique.

Our study establishes the presence of at least three types of secondary vestibulocerebellar neurons in the vestibular nuclei, projecting respectively to the uvulonodular lobule, the flocculus and to both parts of the cerebellum. The number of labeled neurons and their distribution following injections of either fluorescent tracers or HRP (Table 2) are similar. Therefore, the populations of single and double labeled cells may be considered unbiased.

The greatest difference in the number of single fluorescent labeled neurons between individual cases was a factor 2.1 for the uvulo-nodular lobule (exps. K78 and $\mathrm{K} 275$ ) and a factor 1.6 for the flocculus (exps. C825 and K275). Taking into account the different extent of the injection sites and the degree of involvement of the white matter in their central zones, these differences are not excessively large. Naturally, the effect of variation in the individual injections multiplies with respect to the amount of double labeled neurons, in absolute number as well as in percentage. This may be the explaination for the high number of double labeled neurons in case C 825 as well as for the large differences in double labeling between the experiments: e.g. a factor of 6 between experiments $\mathrm{C} 825$ and $\mathrm{K} 275$.

Thus, it is difficult to conclude what is the precise ratio between the three types of vestibulocerebellar neurons. The amount of material is too small to do so and we refrain from a strict interpretation of the cell counts. If the highest number of neurons of each of the three types is taken from Table 2 (regardless of the experiment it comes from) one arrives at a ratio of approximately 12:4:1 (uvulo-nodular lobule single labeled:flocculus single labeled:double labeled).

It remains to be determined how the number of cell types and their ratio will change when other combinations of injection sites are tested (e.g. left FL - right FL). If laterality is taken into account, even with our limited experimental paradigm, the number of possible types increases to five, projecting to: (1) ipsilateral FL, (2) contralateral FL, (3) ipsilateral FL and uvulo-nodular lobule, (4) contralateral FL and uvulo-nodular lobule, and (5) uvulo-nodular lobule.

The distribution of double labeled neurons is very similar to that of neurons projecting to the flocculus. As long as there is no specific knowledge about input on vestibulocerebellar neurons it will be premature to attribute specific functions to the different types. Nevertheless, it should be noted that in the flocculus and the uvulo-nodular lobule the influence of double labeled neurons may differ considerably, since the ratio with single labeled ones may be $1: 4$ for the flocculus and $1: 12$ for the uvulo-nodular lobule. The diversity of responses observed in electrophysiological studies of vestibulocerebellar mossy fibers (Lisberger and Fuchs 1978; Waespe et al. 1981) could find their anatomical substrate in the types of neurons demonstrated here. 
Acknowledgements. The authors wish to thank Erika Goedknegt, Jan Guldemond and Eddie Dalm for their technical assistance during this study, Els de Jong and Paula van Alphen for their assistance during the preparation of the manuscript, and Steve Tan for plotting part of the fluorescent labeled neurons. This investigation was supported by the Foundation for Medical Research, FUN$\mathrm{GO}$, which is subsidized by the Netherlands Organization for the Advancement of Pure Research (Z.W.O.).

\section{References}

Alley K, Baker R, Simpson JI (1975) Afferents to the vestibulocerebellum and the origin of visual climbing fibers in the rabbit. Brain Res 98:582-589

Bentivoglio M, Kuypers HGJM, Catsman-Berrevoets CE (1980a) Retrograde neuronal labeling by means of bisbenzimide and nuclear yellow (Hoechst S 769 121): measures to prevent diffusion of the tracers out of retrogradely labeled neurons. Neurosci Lett 18:19-24

Bentivoglio M, Kuypers HGJM, Catsman-Berrevoets CE, Loewe H, Dann O (1980b) Two new fluorescent retrograde neuronal tracers which are transported over long distances. Neurosci Lett $18: 25-30$

Blanks RHI, Precht W, Torigoe Y (1983) Afferent projections to the cerebellar flocculus in the pigmented rat demonstrated by retrograde transport of horseradish peroxidase. Exp Brain Res 52:293-306

Brodal A (1974) Anatomy of the vestibular nuclei and their connections. In: Kornhuber HH (ed) Handbook of sensory physiology, Vol VI, I. Vestibular system, Part 1. Basic mechanisms. Springer, New York, pp 239-352

Brodal A, Brodal P (1985) Observations on the secondary vestibulocerebellar projections in the macaque monkey. Exp Brain Res 58:62-74

Dow RS (1936) The fiber connections of the posterior parts of the cerebellum in the rat and the cat. J Comp Neurol 63:527-548

Epema AH, Gerrits NM, Voogd J (1988) Commissural and intrinsic connections of the vestibular nuclei in the rabbit: a retrograde tracer study. Exp Brain Res 71:129-146

Gerrits NM (1985) Afferent control of the cerebellum. Thesis. Leiden

Gould BB (1980) Organization of afferents from the brain stem nuclei to the cerebellar cortex in the cat. Adv Anat Embryol Cell Biol $62: 1-79$

Highstein SM, Goldberg J, Moschovakis AK, Fernandez C (1987) Inputs from regularly and irregularly discharging vestibular nerve afferents to secondary neurons in the vestibular nuclei of the squirrel monkey. II. Correlation with output pathways of secondary neurons. J Neurophysiol 58:719-738

Huisman AM, Kuypers HGJM, Verburgh CA (1982) Differences in collateralization of the descending spinal pathways from red nucleus and other brain stem cell group in cat and monkey. In: Kuypers HGJM, Martin GF (eds) Descending pathways to the spinal cord. Progr Brain Res 57:185-219

Ito M (1984) The cerebellum and neural control. Raven Press, New York

Keizer K, Kuypers HGJM, Huisman AM, Dann O (1983) Diamidino yellow dihydrochloride (DY. $2 \mathrm{HCl}$ ): a new fluorescent retrograde neuronal tracer, which migrates only very slowly out of the cell. Exp Brain Res 51: 179-191

Kevetter GA, Mehler WR, Willis WD (1982) Projections to the thalamus from brainstem reticular and vestibular nuclei in the rat. Neurosci Abstr 8: G99

Kotchabhakdi N, Hoddevik GH, Walberg F (1978) Cerebellar afferent projections from the perihypoglossal nuclei: an experimen- tal study with the method of retrograde axonal transport of horseradish peroxidase. Exp Brain Res 31:13-29

Kotchabhakdi N, Walberg F (1978) Cerebellar afferent projections from the vestibular nuclei in the cat: an experimental study with the method of retrograde axonal transport of horseradish peroxidase. Exp Brain Res 31:591--604

Kuypers HGJM, Bentivoglio M, Catsman-Berrevoets CE, Bharos AT (1980) Double retrograde neuronal labeling through divergent axon collaterals, using two fluorescent tracers with the same excitation wavelength which label different features of the cell. Exp Brain Res 40:383-392

Langer T, Fuchs AF, Scudder CA, Chubb MC (1985) Afferents to the flocculus of the cerebellum in the rhesus macaque as revealed by retrograde transport of horseradish peroxidase. J Comp Neurol 235:1-25

Larsell $O(1970)$ The comparative anatomy and histology of the cerebellum from monotremes through apes. The University of Minnesota Press, Minneapolis

Lisberger SG, Fuchs AF (1978) Role of primate flocculus during rapid behavioral modification of vestibulo-ocular reflex. II. Mossy fiber firing patterns during horizontal head rotation and eye movement. J Neurophysiol $41: 764-777$

Magras IN, Voogd J (1985) Distribution of secondary vestibular fibers in the cerebellar cortex: an autoradiographic study in the cat. Acta Anat (Basel) 123:51-57

Mesulam M-M (1982) Tracing neural connections with horseradish peroxidase. IBRO handbook series. Wiley, Chichester

Mitsacos A, Reisine H, Highstein SM (1983) The superior vestibular nucleus: an intracellular HRP study in the cat. II. Non-vestibuloocular neurons. J Comp Neurol 215: 92-107

Nauta HJW, Pritz MB, Lasek RJ (1974) Afferents to the rat caudoputamen studied with horseradish peroxidase: an evaluation of a retrograde neuroanatomical research method. Brain Res $67: 219-238$

Precht W (1979) Vestibular mechanisms. Ann Rev Neurosci 2:265-289

Rosene DL, Mesulam M-M (1978) Fixation variables in horseradish peroxidase neurochemistry. I. The effects of fixation time and perfusion procedures upon enzyme activity. J Histochem Cytochem 26:28-39

Rubertone JA, Haines DE (1981) Secondary vestibulocerebellar projections to flocculonodular lobe in a Prosimian primate, Galago senegalensis. J Comp Neurol 200:255-272

Sato Y, Kawasaki T, Ikarashi K (1983) Afferent projections from the brainstem to the three floccular zones in cats. II. Mossy fiber projections. Brain Res 272:37-48

Thunnissen IE, Epema AH, Gerrits NM (1989) The secondary vestibulocerebellar mossy fiber projection to the caudal vermis in the rabbit. J Comp Neurol 290:262-277

Van Rossum J (1969) Corticonuclear and corticovestibular projections of the cerebellum. Thesis. Van Gorcum, Assen

Voogd J, Feirabend HKP (1981) Classic methods in neuroanatomy. In: Lahue R (ed) Methods in neurobiology, Vol 2. Plenum Press, New York London, pp 301-365

Waespe W, Büttner U, Henn V (1981) Visual-vestibular interaction in the flocculus of the alert monkey. I. Input activity. Exp Brain Res 43:337-348

Walberg F, Dietrichs E (1988) The interconnection between the vestibular nuclei and the nodulus: a study in reciprocity. Brain Res 449:47-53

Yamamoto M (1979) Topographical representation in rabbit cerebellar flocculus for various afferent inputs from the brainstem investigated by means of retrograde transport of horseradish peroxidase. Neurosci Lett 12:29-34

Received May 27, 1989 / Accepted October 16, 1989 